\title{
A Physically Insightful Approach to the Design and Accuracy Assessment of Flux Observers for Field Oriented Induction Machine Drives
}

\author{
Patrick L. Jansen, Student Member, IEEE, and Robert D. Lorenz, Senior Member, IEEE
}

\begin{abstract}
Rotor flux observers can provide an attractive means for achieving direct field oriented control of induction machines. This paper presents a physics-based design methodology and uses it to evaluate open-loop observers and to develop a new closed-loop flux observer. It is shown that the new flux observer is a straightforward structure with properties that combine the best features of known methods. A distinction is made between observers, which use only integration and feedback summation operations, and those estimation methods requiring approximate differentiation which are, in essence, "cancellation" methods. Furthermore, a clear distinction is made between accuracy and dynamic robustness of the observer. This distinction is important because the accuracy of flux observers for induction machines is inherently parameter sensitive. Whereas robustness of observers, in a controls sense, is not parameter sensitive. Moreover, it is shown how flux observers can provide robust field oriented control because the flux angle is substantially more correct than the flux magnitude. A distinctive form of frequency response function (FRF) analysis similar to that used in classical control engineering is demonstrated to be a useful and insightful tool even though flux observers are multiple-input, multiple-output systems. Finally, the limits of such flux observers are experimentally evaluated.
\end{abstract}

\section{INTRODUCTION}

$\mathbf{T}$

THERE ARE two basic forms of rotor flux field orientation; direct field orientation which relies on direct measurement or estimation of the rotor flux magnitude and angle and indirect field orientation which utilizes an inherent slip relation [1]. Because indirect field orientation is essentially a feedforward scheme it is naturally parameter sensitive, particularly to the rotor time constant, and thus has led to the development of numerous parameter adaption schemes [2].

The implementation of direct field orientation via air gap flux measurement has typically been plagued by the complexities and lack of mechanical robustness associated with intrusive sensors located within machine air gaps. Furthermore, a correction is required for the rotor leakage flux if rotor flux field orientation is to be achieved. However, a recently demonstrated method of measuring the flux angle via the saturation-induced third harmonic in the phase voltages is promising as it alleviates the need of intrusive sensors or modifications of the machine. [3]

Paper MA 1-94, approved by the Industrial Drives Committee of the IEEE Industry Applications Society for presentation at the 1992 Industry Applications Society Annual Meeting, Houston, TX, October 4-9.

The authors are with the Dept. of Electrical and Computer Engineering, University of Wisconsin-Madison, Madison, WI 53706.

IEEE Log Number 9213418.
Estimation rather than measurement of the rotor flux is an alternative approach for direct field orientation that has received considerable attention. [4-13] As with the third harmonic scheme, only terminal properties measurement (in some cases including rotor speed) is required. However, the accuracy associated with such estimation schemes is inherently parameter sensitive. The degree of sensitivity to both machine parameter estimates and also measured signal noise, e.g. quantization noise, filter attributes, sample rates, etc., is dependent upon the particular estimation scheme that is implemented.

Even though observers in general have been around for several decades [14-15], a clear and physically-insightful method of developing flux observers has not yet evolved. Furthermore, an insightful method for evaluating the accuracy implications of parameter errors for applications such as rotor flux estimation is still needed. This lack is perhaps a fundamental problem with the current methodology of designing observers based solely upon Gopinath's minimal order observer theory [14]. This paper attempts to present in a straightforward manner the accuracy attributes of both open and closed-loop nonlinear rotor flux observers for direct field orientation. It attempts to lay a foundation for a physics-based design and accuracy analysis methodology applicable to both linear and nonlinear observers.

Technically, the word "observer" implies an estimator that employs both inputs to integration process models and feedback control for error correction to improve estimation accuracy and for defining the error correction dynamics. In this paper, such estimators with feedback correction controls are denoted "closed-loop observers", while "open-loop observers" refer to estimators that employ integration process models without feedback correction. The open-loop observer is then essentially a real-time simulation of the physical process, i.e. the induction machine in this case. As such, one important property of the open-loop observer is that it provides nearly instantaneous tracking capability. It will be shown how the closed-loop observer can be formed to inherently retain this nearly instantaneous tracking capability.

The third form of estimator evaluated in this paper is based on cancellation methodologies, whereby approximate differentiation of signals is used to cancel the effects of process integration. In linear systems, this approach is also known as pole/zero cancellation. However, differentiation makes such approaches susceptible to measurement and quantization noise. 


\section{COMPLEX Vector INDUCtion MACHINE MODEL}

The analysis of observers for symmetric induction machines can be simplified considerably by the use of complex vector notation. Complex vector notation reduces the order of the system by a factor of two and also simplifies the cross coupling between the $q$ and $d$ axes through the use of the inherent $90^{\circ}$ phase shift provided by $j$. It thus allows the treatment of the rotor flux as a single vector quantity allowing the development of frequency response functions (FRF). In general, the complex quantities are written in the form, $\mathrm{f}_{q d s} \equiv$ $f_{q s}-j f_{d s}$.

For implementing direct rotor flux field orientation, the stationary reference is the most suitable. Therefore, with the stationary reference implied and all rotor quantities referred to the stator, the induction machine electrical dynamics are characterized by the following equations:

$$
\begin{aligned}
\mathbf{v}_{q d s} & =r_{s} \mathbf{i}_{q d s}+p \lambda_{q d s} \\
0 & =r_{r} \mathbf{i}_{q d r}+\left(p-j w_{r}\right) \lambda_{q d r}
\end{aligned}
$$

where $p$ is the differential operator and the flux linkages are

$$
\begin{aligned}
& \lambda_{q d s}=L_{s} \mathbf{i}_{q d s}+L_{m} \mathbf{i}_{q d r} \\
& \lambda_{q d r}=L_{r} \mathbf{i}_{q d r}+L_{m} \mathbf{i}_{q d s}
\end{aligned}
$$

In addition, it will prove useful to define the following complex impedances:

$$
\begin{aligned}
& \mathbf{Z}_{s} \equiv r_{s}+\sigma L_{s} p \\
& \mathbf{Z}_{s}^{\prime} \equiv r_{s}^{\prime}+\sigma L_{s} p \\
& \mathbf{Z}_{r} \equiv L_{r}\left(p+\omega_{b r}\right)
\end{aligned}
$$

where $\sigma$ is the leakage or coupling factor,

$$
\begin{aligned}
\sigma & \equiv 1-\frac{L_{m^{2}}}{L_{r} L_{s}}, \\
r_{s}^{\prime} & \equiv r_{s}+r_{r} \frac{L_{m^{2}}}{L_{r^{2}}},
\end{aligned}
$$

and

$$
\omega_{b r} \equiv \frac{r_{r}}{L_{r}}-j \omega_{r}
$$

Note that $\mathbf{Z}_{s}^{\prime}$ is a stator transient impedance and $\omega_{r}$ is the rotor velocity.

Equations (1-4) can be rearranged in terms of the stator current and rotor flux in a form similar to a state space model with the current and flux being the state variables. Note, however, the system is nonlinear with respect to rotor velocity.

$$
\begin{aligned}
p \mathbf{i}_{q d s} & =\frac{1}{\sigma L_{s}}\left(\mathbf{v}_{q d s}-r_{s}^{\prime} \mathbf{i}_{q d s}+\frac{L_{m}}{L_{r}} \omega_{b r} \lambda_{q d r}\right) \\
p \lambda_{q d r} & =\frac{r_{r}}{L_{r}} L_{m} \mathbf{i}_{q d s}-\omega_{b r} \lambda_{q d r}
\end{aligned}
$$

From (11) and (12), the induction machine electrical model can be formulated into a nonlinear block diagram as shown in Fig. 1 with stator voltage and rotor velocity treated as system inputs.

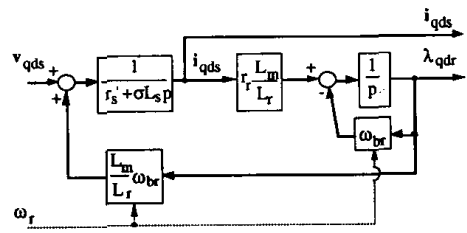

Fig. 1. Induction machine electrical model based upon stator current and rotor flux as state variables and stator voltage and rotor velocity as system inputs. (in stationary reference, using complex vector notation, the dashed line denotes a functional dependence on $\omega_{r}$ ).

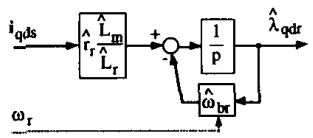

Fig. 2. Open loop rotor flux observer based upon the current model utilizing measured stator current and rotor velocity. (in stationary reference, using complex vector notation, ` denotes estimated quantities, dashed line denotes functional dependence on $\omega_{r}$ ).

\section{OPEN-LOOP FluX OBSERVERS \& ESTIMATORS}

There are three basic topologies for open-loop flux observers as dictated by the measured states/inputs and corresponding flux model. Two are reduced order models that have been referred to as the current and the voltage models [5]; with a third observer being essentially a full order model with respect to the induction machine electrical model. In addition there is an estimator based on cancellation methods.

\section{A. Current Model Open-Loop Flux Observers}

From (12) and Fig. 1, it is apparent that an open-loop rotor flux observer can be formed if the stator current and the rotor velocity are measured in real time. From machine parameter estimates (denoted by ${ }^{\wedge}$ ), the governing equation of the open-loop rotor flux observer is:

$$
p \hat{\lambda}_{q d r}=\hat{r}_{r} \frac{\hat{L}_{m}}{\hat{L}_{r}} \mathbf{i}_{q d s}-\hat{\omega}_{b r} \hat{\lambda}_{q d r}
$$

A block diagram of this current model based observer is shown in Fig. 2.

Unfortunately, the parameter estimates used within the observer are never exactly correct. To evaluate the parameter dependent accuracy of the estimated flux, a FRF relating the estimated and actual fluxes has been found to be insightful and helpful. From (12) and (13), the estimated flux can be expressed as a response to the actual flux;

$$
\begin{aligned}
\frac{\hat{\lambda}_{q d r}}{\lambda_{q d r}} & =\frac{\left(\hat{r}_{r} \frac{\hat{L}_{m}}{\hat{L}_{r}}\right)}{\left(r_{r} \frac{L_{m}}{L_{r}}\right)} \frac{\left(p+\omega_{b r}\right)}{\left(p+\hat{\omega}_{b r}\right)} \\
& =\frac{\hat{r}_{r} \hat{L}_{m}}{r_{r} L_{m}} \frac{\mathbf{Z}_{r}}{\hat{\mathbf{Z}}_{r}}=\mathrm{FRF}_{C}
\end{aligned}
$$

For steady state operation, $p$ may be replaced by the excitation frequency, $j \omega_{e}$, and the response function can be expressed as 

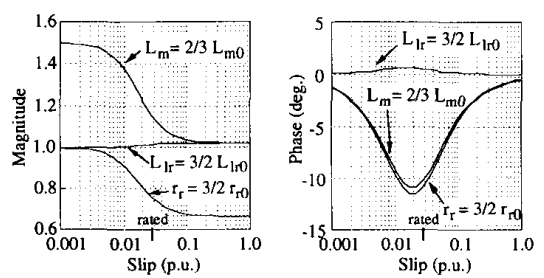

Fig. 3. $\hat{\lambda}_{q d r} / \lambda_{q d r}$, FRF (15): Current model open-loop rotor flux observer with machine parameter deviations from nominal $r_{r 0}, L_{m 0}$, and $L_{l r 0}$.

a function of the slip frequency, $\omega_{s}$, where $\omega_{s}=\omega_{e}-\omega_{r}$

$$
\begin{aligned}
\frac{\hat{\lambda}_{q d r}}{\lambda_{q d r}} & =\frac{\left(\hat{r}_{r} \frac{\hat{L}_{m}}{\hat{L}_{r}}\right)}{\left(r_{r} \frac{L_{m}}{L_{r}}\right)} \frac{\left(\frac{r_{r}}{L_{r}}+j \omega_{s}\right)}{\left(\frac{\hat{r}_{r}}{\hat{L}_{r}}+j \omega_{s}\right)} \\
& =\frac{\hat{L}_{m}}{L_{m}} \frac{\left(1+j \frac{L_{r}}{r_{r}} \omega_{s}\right)}{\left(1+j \frac{\hat{L}_{r}}{\hat{r}_{r}} \omega_{s}\right)}
\end{aligned}
$$

The influence of parameter estimate errors on the accuracy of the estimated flux is evident in the FRF magnitude and phase plots of (15) in Fig. 3. At high slip the rotor flux magnitude response is sensitive primarily to the rotor resistance, while the estimated rotor flux phase angle is very insensitive to all parameter estimates. Near rated slip, both the flux angle and magnitude are sensitive to the estimated rotor resistance and magnetizing inductance. Note the accuracy is relatively unaffected by the rotor leakage inductance for any operating condition.

\section{B. Voltage Model Open-Loop Flux Observers}

The voltage model utilizes the measured stator voltages and currents, but not the rotor velocity. It is commonly used to implement direct field orientation without measured velocity feedback for low cost drive applications $[2,16]$. From (1), the stator flux can be estimated by integration of the following equation,

$$
p \hat{\lambda}_{q d s}=\mathbf{v}_{q d s}-\hat{r}_{s} \mathbf{i}_{q d s}
$$

from which the rotor flux is then obtained using (3) \& (4),

$$
\hat{\lambda}_{q d r}=\frac{\hat{L}_{r}}{\hat{L}_{m}} \hat{\lambda}_{q d s}-\frac{\hat{\sigma}}{1-\hat{\sigma}} \hat{L}_{m} \mathbf{i}_{q d s}
$$

A block diagram for the observer is shown in Fig. 4. Since the voltage model is essentially an integrator without feedback, it is sensitive to offset and drift errors. Thus a major problem with this open-loop observer is that it lacks feedback necessary for convergence. In practice, a low pass integrator is often used to provide stability.

The corresponding flux estimation FRF is:

$\frac{\hat{\lambda}_{q d r}}{\lambda_{q d r}}=\frac{L_{m} \hat{L}_{r}}{\hat{L}_{m} L_{r}}\left[1+\frac{1}{r_{r}} \frac{L_{r}^{2}}{L_{m}^{2}}\left(\frac{p+\omega_{b r}}{p}\right)\left(\mathbf{Z}_{s}-\hat{\mathbf{Z}}_{s}\right)\right]=\mathrm{FRF}_{V}$.

Replacing $p$ by the excitation frequency, $j \omega_{e}$, and incorporating the slip frequency, $\omega_{s}$, the voltage model flux estimation

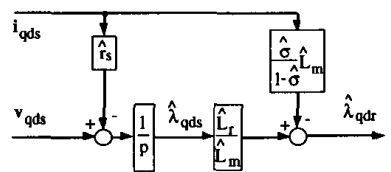

Fig. 4. Open loop rotor flux observer based upon the voltage model utilizing measured stator voltages and currents (in stationary reference, using complex vector notation)
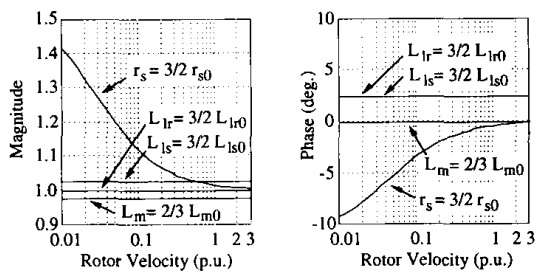

Fig. 5. $\hat{\lambda}_{q d r} / \lambda_{q d r}$, FRF (19): Voltage model open-loop rotor flux observer at rated slip with machine parameter deviations from nominal $r_{s 0}, L_{m 0}, L_{l s 0}$, and $L_{\text {Iro }}$.

FRF can be rewritten as

$$
\begin{aligned}
& \frac{\hat{\lambda}_{q d r}}{\lambda_{q d r}}=\frac{L_{m} \hat{L}_{r}}{\hat{L}_{m} L_{r}}\left[1+\frac{1 L_{r}^{2}}{r_{r} L_{m}^{2}}\left(\frac{r_{r}}{L_{r}}+j \omega_{s}\right)\right. \\
& \left.\left(\left(\sigma L_{s}-\hat{\sigma} \hat{L}_{s}\right)-j \frac{\left(r_{s}-\hat{r}_{s}\right)}{\left(\omega_{s}+\omega_{r}\right)}\right)\right]
\end{aligned}
$$

With field oriented control, the slip frequency is held constant for given torque and flux commands independent of rotor velocity. Thus, analysis of the observer accuracy under this operating condition is very appropriate. Magnitude and phase plots of the complete FRF (19) are shown in Fig. 5 at rated slip frequency and variable velocity with parameter deviations from nominal.

Unlike the current model based observer, the accuracy of this observer is completely insensitive to rotor resistance but is most sensitive to stator resistance at low rotor velocities. At higher rotor velocities, the stator resistance IR drop in (16) is less significant relative to the speed voltage (back emf), resulting in reduced sensitivity to stator resistance. However, the low speed sensitivity is a well acknowledged limitation of this observer $[2,16]$.

Because the attributes of the two open-loop observers are in many ways complementary, there has been at least one attempt to create a better observer by directly merging the two models via selected weighting [4]. The concept is well merited however a better approach will be found in the form of a closed-loop observer.

\section{Full Order Open-Loop Flux Observers}

The full order open-loop flux observer (with respect to the machine electrical model) is derived directly from the induction machine electrical model characterized by (11) and (12) and is depicted in Fig. 6. The observer utilizes measured stator voltage and rotor velocity. The stator current is estimated as an intermediary quantity only. 


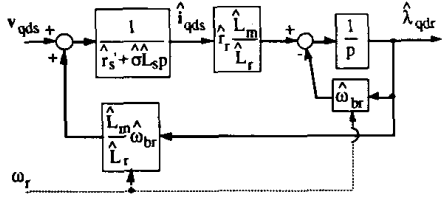

Fig. 6. Open-loop rotor flux observer based upon the full order induction machine electrical model utilizing measured stator voltages and rotor velocity-(in stationary reference, using complex vector notation).
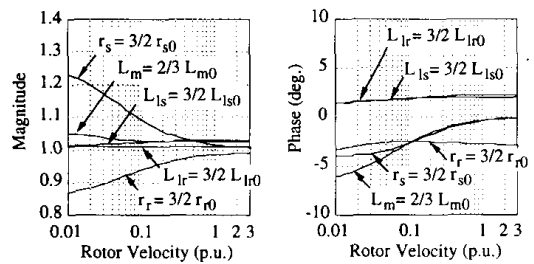

Fig. 7. $\hat{\lambda}_{q d r} / \lambda_{q d r}$, FRF (21): Full order model open-loop rotor flux observer at rated slip with machine parameter deviations from nominal $r_{r 0}, r_{s 0}, L_{m 0}, L_{l s 0}$ and $L_{l r 0}$.

The corresponding FRF can be written:

$$
\frac{\hat{\lambda}_{q d r}}{\lambda_{q d r}}=\frac{\frac{\mathbf{Z}_{s} \mathbf{Z}_{r}}{r_{r} L_{m}}+\frac{L_{m}}{L_{r}} p}{\frac{\hat{\mathbf{Z}}_{s} \hat{\mathbf{Z}}_{r}}{\hat{r}_{r} \hat{L}_{m}}+\frac{\hat{L}_{m}}{\hat{L}_{r}} p}=\frac{\mathrm{FRF}_{F n}}{\mathrm{FRF}_{F d}}=\mathrm{FRF}_{F}
$$

Under steady state conditions, $p=j \omega_{e}$ and (20) can then be written as:

$$
\frac{\hat{\lambda}_{q d r}}{\lambda_{q d r}}=\frac{\left[\frac{r_{s}}{L_{m}}-\left(\frac{\sigma}{1-\sigma}\right) \frac{L_{m}}{r_{r}} \omega_{s} \omega_{e}\right]+j\left[\frac{r_{s} L_{r}}{r_{r} L_{m}} \omega_{s}+\frac{L_{s}}{L_{m}} \omega_{e}\right]}{\left[\frac{\hat{r}_{s}}{\hat{L}_{m}}-\left(\frac{\hat{\sigma}}{1-\hat{\sigma}}\right) \frac{\hat{L}_{m}}{\hat{r}_{r}} \omega_{s} \omega_{e}\right]+j\left[\frac{\hat{r}_{s} \hat{L}_{r}}{\hat{r}_{r} \hat{L}_{m}} \omega_{s}+\frac{\hat{L}_{s}}{\hat{L}_{m}} \omega_{e}\right]}
$$

As illustrated by the FRF magnitude and phase plots in Fig. 7 at rated slip, a similarity to the voltage model exists; the sensitivity to stator resistance decreases with increasing velocity, and the leakage inductance sensistivity is relatively small but nearly constant.

Likewise, some semblance to the current model is also evident. At high speeds, such that $\omega_{e} \gg \omega_{s}$, the FRF given by (21) can be approximated by:

$$
\frac{\hat{\lambda}_{q d r}}{\lambda_{q d r}} \approx \frac{\frac{L_{s}}{L_{m}}\left(1+j \frac{\sigma L_{r}}{r_{r}} \omega_{s}\right)}{\frac{\hat{L}_{s}}{\hat{L}_{m}}\left(1+j \frac{\hat{\sigma} \hat{L}_{r}}{\hat{r}_{r}} \omega_{s}\right)}
$$

Equation (22) is similar in form to the current model FRF given by (15), although the presence of the leakage factor $(\sigma)$ has moved the slip corresponding to maximum sensitivity to much higher than rated. Furthermore, the sensitivity to magnetizing inductance $\left(L_{m}\right)$ is drastically reduced. These conclusions are illustrated by the FRF plots of (21) in Fig. 8 taken at rated excitation, $\omega_{e}$ and variable slip.

Although the parameter sensitivity is not unattractive, the need for accurately measured voltages at low and zero speeds (like the voltage model) is anticipated to be a significant limitation for this observer. Furthermore, the observer dynamics are considerably more complex than the voltage and current models.
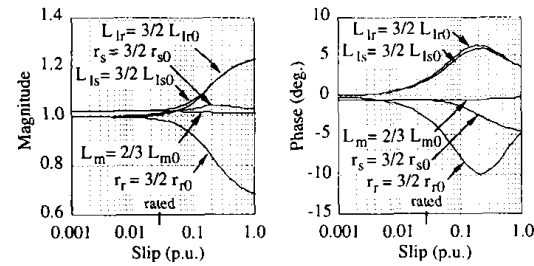

Fig. 8. $\hat{\lambda}_{q d r} / \lambda_{q d r}$, FRF (21): Full order model open-loop rotor flux observer at rated excitation $\left(\omega_{e}=2 \pi 60\right)$ with machine parameter deviations from nominal $r_{r 0}, r_{s 0}, L_{m 0}, L_{l s 0}$, and $L_{l r 0}$.

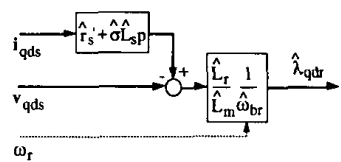

Fig. 9. Open loop rotor flux estimator based upon the cancellation method utilizing measured stator voltage and current and rotor velocity. (in stationary reference, using complex vector notation).
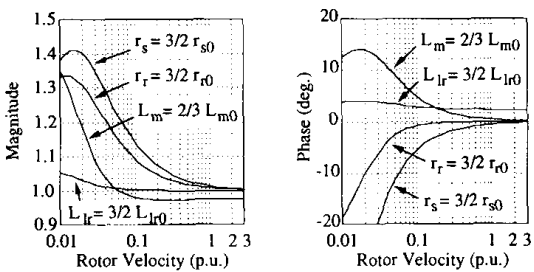

Fig. 10. $\hat{\lambda}_{q d r} / \lambda_{q d r}$, FRF (24): FRF of open-loop, cancellation method flux estimator at rated slip with machine parameter deviations from nominal $r_{s 0}, r_{r 0}, L_{m 0}$, and $L_{l r 0}$

\section{Cancellation Method Open-Loop Flux Estimators}

It is possible to construct a cancellation method open-loop flux estimator based on the rotor and stator flux linkage and voltage loop equations as combined in (11) after substituting the estimated machine parameters and measured terminal properties as follows.

$$
\hat{\lambda}_{q d r}=\frac{\hat{L}_{r}}{\hat{L}_{m}} \frac{1}{\hat{\omega}_{b r}}\left[-\mathbf{v}_{q d s}+\left(\hat{r}_{s}^{\prime}+\hat{\sigma} \hat{L}_{s} p\right) \mathbf{i}_{q d s}\right]
$$

Noting the requirement for stator current differentiation, this cancellation method estimator would then appear as in Fig. 9.

The estimation FRF for the open loop cancellation method is obtained by solving (11), (12), and (23) simultaneously to achieve

$$
\frac{\hat{\lambda}_{q d r}}{\lambda_{q d r}}=\frac{\frac{L_{m}}{L_{r}} \omega_{b r}+\frac{\mathbf{Z}_{r}}{r_{r} L_{m}}\left(\hat{\mathbf{Z}}_{s}^{\prime}-\mathbf{Z}_{s}^{\prime}\right)}{\frac{\hat{L}_{m}}{\hat{L}_{r}} \hat{\omega}_{b r}}=\mathrm{FRF}_{E}
$$

The accuracy of this method is depicted in the FRF plot of Fig. 10 for rated slip operation of the machine.

The inherently high parameter sensitivity of this method is readily apparent from the various deviations shown in Fig. 10. This method is also especially problematic due to the division by $\hat{\omega}_{b r}$ when the rotor velocity is near zero. In this range the quantization noise due to numerical differentiation of such signals to obtain rotor velocity typical of modern digital 


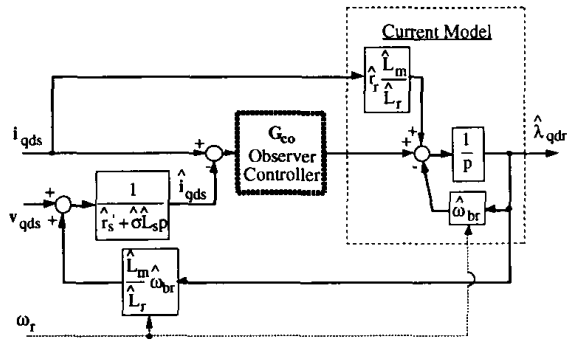

Fig. 11. A closed-loop, Gopinath style rotor flux observer for an induction machine based upon the current model and utilizing corrective current error feedback.

position feedback drive systems becomes a dominant form of contamination for the estimate.

After evaluating the parameter dependent accuracy of the various open-loop approaches, it now is appropriate to evaluate what accuracy improvements can be gained by forming a closed-loop observer.

\section{Closed-Loop Flux ObServers}

A closed-loop rotor flux observer from the current model open-loop observer in Fig. 2 can be developed directly from the induction machine model of Fig. 1. Measuring the stator voltage allows the stator current to be estimated and compared to the measured current as shown in Fig. 11. Because the rotor flux acts as a form of physical state feedback on the stator current, the current error can be driven to zero through the use of corrective feedback acting on the estimated rotor flux. Ideally the rotor flux estimate will converge on the correct value when the current error goes to zero.

Because the observer in Fig. 11 is loosely based upon Gopinath's minimal order observer theory, it will be referred to as a Gopinath style observer in this paper. [14] The primary differences between the observer in Fig. 11 and a true Gopinath minimal order observer are the assumed generality of the controller, $\mathbf{G}_{c o}$, and the estimated transient resistance voltage drop. Gopinath's observer utilizes the measured current, i.e. $\hat{r}_{s}^{\prime} \mathbf{i}_{q d s}$, rather than the estimated current as in Fig. 11, i.e. $\hat{r}_{s}^{\prime} \hat{\mathbf{i}}_{q d s}$. The difference in parameter sensitivity can be shown to be negligible for this particular observer.

A full order closed-loop observer can be constructed that incorporates corrective feedback acting on both the rotor flux and stator current estimates as shown in Fig. 12. [6]

Although topologically similar, controllers used for closedloop observers differ from physical system controllers. One important aspect is that no physical energy transfer occurs within the observer. Hence, the gains and the eigenvalues are not limited by physical constraints, but rather by disturbances in the form of measured signal quantization noise, and measured signal harmonic noise.

Another significant distinction between closed-loop observers and closed-loop physical systems lies in the properties of robustness and accuracy.

For the closed-loop observer, the model parameters and the observer controller's gains are fixed and thus entirely known by the controls designer. Thus, the closed-loop eigenvalues of

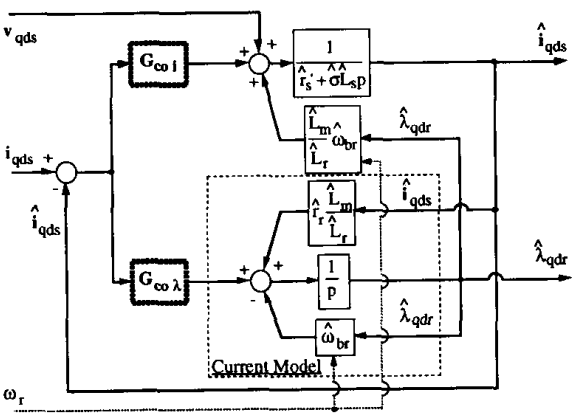

Fig. 12. A full order rotor flux and stator current closed-loop observer.

all observers are completely deterministic, and the robustness of a closed-loop observer is guaranteed automatically by design.

For physical systems, only the controller's gains are entirely known and fixed by the controls designer. The plant model topology and its parameters are estimates, and should not be assumed to be correct. Thus, the closed-loop eigenvalues of controlled physical systems are not completely deterministic, and the robustness of the closed-loop physical system is not guaranteed automatically by design.

For the closed-loop observer, the accuracy of the estimates is determined by the accuracy of the open-loop observer model, by the observer topology (determined by which variables/inputs are measured and which are estimated), and by the observer controller's gains. Thus, a closed-loop observer is not inherently accurate in estimating either static or dynamic properties. The following discussion of the closed-loop observer from Fig. 8 and alternative topologies will provide analysis to demonstrate such properties.

By comparison, the accuracy of a closed-loop physical system is primarily determined by the accuracy of the measured variables (sensor and signal conditioning accuracy) and only secondarily by the physical system controller in the way the controller rejects disturbances. Thus, a closed loop physical system is deterministically made accurate.

It should be noted that the accuracy of the closed-loop observer is critical as it determines the robustness of the observer-based control system, e.g. the field oriented induction machine controller.

\section{A. Implicit Flux References for Closed-Loop Observers}

To evaluate the accuracy of the Gopinath style closed-loop observer, the model of Fig. 11 may be modified by simple block diagram algebra of the inputs and feedback to show the closed loop flux estimation as in Fig. 13.

This analysis shows that the implicit flux reference, $\hat{\lambda}_{q d r}^{*}$, for this general Gopinath style flux observer is produced by an open-loop cancellation method estimator. This is a particularly critical finding since it implies that even if the observer controller tracks the reference perfectly, the observer's flux estimate will still be sensitive to parameter errors. Furthermore, at frequencies above the observer bandwidth the observer will 


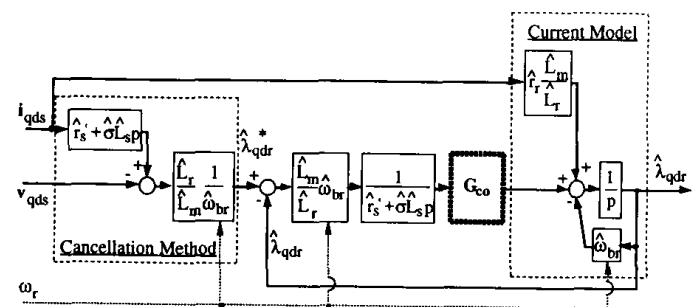

Fig. 13. Closed-loop, Gopinath style flux observer after block diagram algebraic manipulation to show the "implicit" flux estimation reference, $\hat{\lambda}_{q d r}^{*}$ " as produced by an open loop cancellation method estimator.

follow the error properties of the current model open-loop observer.

If the controller $\mathbf{G}_{c o}$ is assumed to be a linear controller with complex gain $\mathbf{K}$, the Gopinath style observer is characterized by the equations:

$$
\begin{aligned}
p \hat{\mathbf{i}}_{q d s} & =\frac{1}{\hat{\sigma} \hat{L}_{s}}\left(\mathbf{v}_{q d s}-\hat{r}_{s}^{\prime} \hat{\mathbf{i}}_{q d s}+\frac{\hat{L}_{m}}{\hat{L}_{r}} \hat{\omega}_{b r} \hat{\lambda}_{q d r}\right) \\
p \hat{\lambda}_{q d r} & =\hat{r}_{r} \frac{\hat{L}_{m}}{\hat{L}_{r}} \mathbf{i}_{q d s}-\hat{\omega}_{b r} \hat{\lambda}_{q d r}+\mathbf{K}\left(\mathbf{i}_{q d s}-\hat{\mathbf{i}}_{q d s}\right)
\end{aligned}
$$

The corresponding FRF is then given by (27) which clearly illustrates the presence of the cancellation method as an implicit flux reference.

$$
\frac{\hat{\lambda}_{q d r}}{\lambda_{q d r}}=\frac{\frac{\hat{\mathbf{Z}}_{r} \hat{\mathbf{Z}}_{s}^{\prime}}{\hat{L}_{m} \hat{\omega}_{b r}}\left(\mathrm{FRF}_{C}\right)+\mathbf{K}\left(\mathrm{FRF}_{E}\right)}{\frac{\hat{\mathbf{Z}}_{r} \hat{\mathbf{Z}}_{s}^{\prime}}{\hat{L}_{m} \hat{\omega}_{b r}}+\mathbf{K}}
$$

Assuming linear controllers $\mathbf{K}_{\lambda}$ and $\mathbf{K}_{i}$, the full order closed-loop observer is characterized by (28) and (29).

$$
\begin{aligned}
p \hat{i}_{q d s}= & \frac{1}{\hat{\sigma} \hat{L}_{s}}\left(\mathbf{v}_{q d s}-\hat{r}_{s}^{\prime} \hat{\mathbf{i}}_{q d s}\right. \\
& \left.+\frac{\hat{L}_{m}}{\hat{L}_{r}} \hat{\omega}_{b r} \hat{\lambda}_{q d r}\right)+\mathbf{K}_{i}\left(\mathbf{i}_{q d s}-\hat{\mathbf{i}}_{q d s}\right) \\
p \hat{\lambda}_{q d r}= & \hat{r}_{r} \frac{\hat{L}_{m}}{\hat{L}_{r}} \mathbf{i}_{q d s}-\hat{\omega}_{b r} \hat{\lambda}_{q d r} \\
& +\mathbf{K}_{\lambda}\left(\mathbf{i}_{q d s}-\hat{\mathbf{i}}_{q d s}\right)
\end{aligned}
$$

Although similar implicit flux references can not be easily identified within the full order closed-loop observer via simple block diagram algebra, the corresponding FRF in (30) clearly identifies three flux references corresponding to the current model, the full order open-loop observer, and the cancellation method estimator.

$$
\frac{\hat{\lambda}_{q d r}}{\lambda_{q d r}}=\frac{\left(\mathrm{FRF}_{F n}\right)+\mathbf{K}_{\lambda} \hat{L}_{m} \hat{\omega}_{b r}\left(\mathrm{FRF}_{E}\right)+\mathbf{K}_{i} \hat{\mathbf{Z}}_{r}\left(\mathrm{FRF}_{C}\right)}{\left(\mathbf{F R F _ { F d }}\right)+\mathbf{K}_{\lambda} \hat{L}_{m} \hat{\omega}_{b r}+\mathbf{K}_{i} \hat{\mathbf{Z}}_{r}}
$$

Thus depending upon the relative gain selection (eigenvalue placement), the observer's flux estimate will be sensitive to the parameters of the cancellation method estimator and/or the

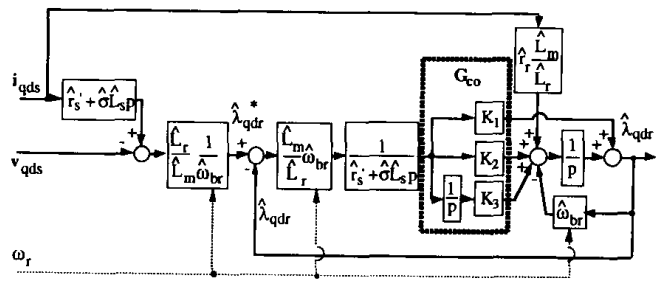

Fig. 14. Closed-loop Gopinath style flux observer with speed varying dynamics due to linear feedback gains and nonlinear flux model.

current model. At frequencies beyond the observer bandwidth, the observer will be sensitive to the full order open-loop observer.

It should be noted that these conclusions are not dependent on the actual configuration (sliding mode vs. PID, nonlinear, etc.) of the observer controller. However, alternative controllers cause different estimation dynamics and transition regions between the open-loop models. Thus, it is instructive to see how alternative closed loop observer controller topologies can affect design and transition properties. Both linear gain and nonlinear gain observer controllers will be evaluated.

\section{B. Linear Gain Gopinath Observer Controllers}

Fig. 14 shows a linear feedback gain controller for a Gopinath style, closed-loop flux observer. Because the closedloop observer is a controlled system within itself, the utilization of a set of controller gains including an integral control action is appropriate to deterministically set the observer's dynamic and static properties. The observer's dynamics are governed by three pairs of complex eigenvalues that vary with velocity. Such state variant dynamics are common to all nonlinear systems if constant controller gains are used.

It should be noted that this observer is particularly tedious to tune due to the large, nonlinear changes in its dynamics as a function of rotor velocity. Thus, despite the deterministic nature of observers, the nonlinearities included in the observer make this implementation impractical. The next section develops an intrinsically robust, velocity invariant design methodology which lends itself to practical implementation.

\section{Nonlinear, Velocity Invariant Observer Controllers}

The nonlinear, velocity varying dynamics of the flux observer can be made velocity invariant by different observer controller design approaches. The classical approach taken by prior researchers has been to form velocity dependent gain terms via algebraic eigenvalue solution techniques [8]. In this section an alternative approach is presented based on techniques which allow for substantial insight in how nonlinear physical system dynamics may be altered by controls design to achieve invariant closed loop dynamics $[17,18]$.

Fig. 15 introduces such a nonlinear observer controller alternative which considerably reduces the tuning complexity for the Gopinath style flux observer.

This controller achieves velocity invariant (fixed) dynamics by using a combination of three nonlinear design techniques: nonlinear state feedback decoupling of $\hat{\omega}_{b r} \hat{\lambda}_{q d r}$, nonlinear 


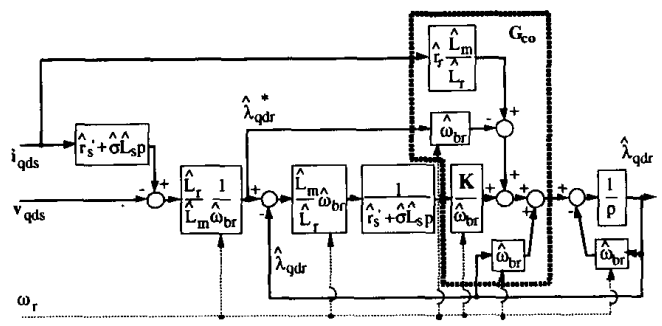

Fig. 15. Closed-loop Gopinath style flux observer with velocity invariant dynamics due to the nonlinear controller for the nonlinear flux model.

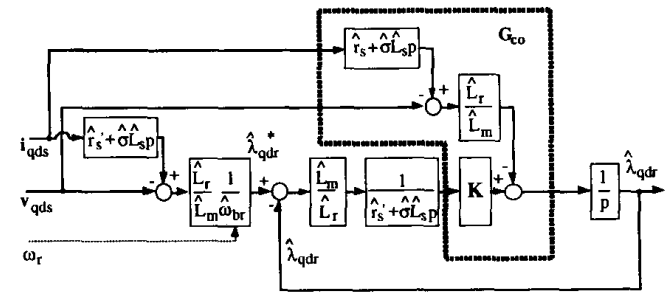

Fig. 16. Closed-loop Gopinath style flux observer with velocity invarian dynamics-showing implementation terms which have been simplified.

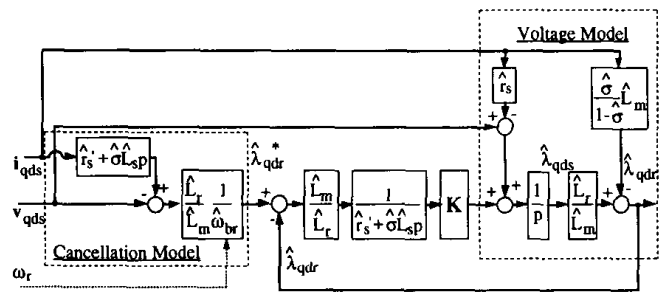

Fig. 17. Closed-loop Gopinath flux observer with speed invariant error correction dynamics-showing both cancellation model flux reference and voltage models as the equivalent observer model basis.

command feedforward of $-\hat{\omega}_{b r} \hat{\lambda}_{q d r}$, and nonlinear manipulated input decoupling of $1 / \hat{\omega}_{b r}$, along with the linear state feedback controller, $\mathbf{K}[17,18]$.

In configuring the controller, the feedforward estimation model is set to retain the estimation properties of the current model open-loop observer. Thus, the nonlinear state feedback decoupling term requires that a corresponding term be added to the command feedforward controller. The manipulated input decoupling is set to remove the velocity varying nature of the forward loop elements in the closed-loop observer model.

Because this entire observer model is internal to the control processor, it is possible to algebraically simplify the observer implementation. Fig. 16 shows the result after simplification of feedback and feedforward terms.

Insight on the velocity invariant observer is enhanced by forming the feedforward terms to avoid differentiation, resulting in an explicit voltage model shown in Fig. 17.

This observer formulation may be further simplified by realizing that the first two terms after the flux summing junction

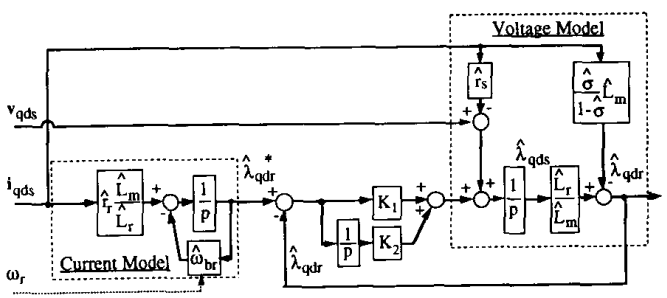

Fig. 18. Closed-loop Gopinath style flux observer with speed invariant error correction dynamics-showing implementation with simplified controller and current model input acting as implicit reference.
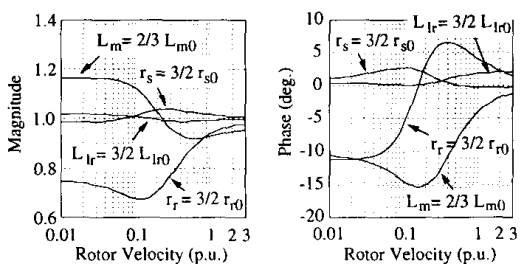

Fig. 19. $\hat{\lambda}_{q d r} / \lambda_{q d r}$ FRF (31): Nonlinear feedback gain, closed-loop, Gopinath style flux observer with rated slip. (Eigenvalues $-\sigma_{r} 1=-\sigma_{r} 2=2 \pi 10 \mathrm{rad} / \mathrm{sec}$ ) and with machine parameter deviations from nominal $r_{s 0}, r_{r 0}, L_{m 0}$, and $L_{l r 0}$.

are not needed to retain the accuracy of the model. Removing the low pass term will allow a simpler controller configuration. Furthermore, the implicit flux reference requirement can also be met by replacing the cancellation model flux estimator with the more desirable current model open-loop flux observer. The final configuration is shown in Fig. 18.

The resulting closed-loop flux observer is seen to provide an automatic transition between the two most desirable open-loop flux observer models: the current model at low frequencies to the voltage model at high frequencies. This transition is now determined via the bandwidth of the flux loop which is velocity invariant and easy to tune.

The FRF of flux estimation for this observer has the following form:

$$
\frac{\hat{\lambda}_{q d r}}{\hat{\lambda}_{q d r}}=\frac{\frac{\hat{L}_{m}}{\hat{L}_{r}} p\left(\mathbf{F R F}_{V}\right)+\mathbf{K}\left(\mathrm{FRF}_{C}\right)}{\frac{\hat{L}_{m}}{\hat{L}_{r}} p+\mathbf{K}}
$$

which is shown graphically in Fig. 19.

The FRF plots show how this topology combines the best of both models and thus demonstrates combined accuracy attributes. Furthermore, this form does not have the noise sensitivity of the cancellation method.

\section{IMPLEMENTATION OF OBSERVER-BASED DIRECT FIELD ORIENTATION}

Although the estimation dynamics of the closed-loop observer in Fig. 18 are velocity invariant, the open-loop current model dynamics as implemented in the stationary frame are not. The velocity variant cross coupling due to $\hat{\omega}_{b r}$ results in 


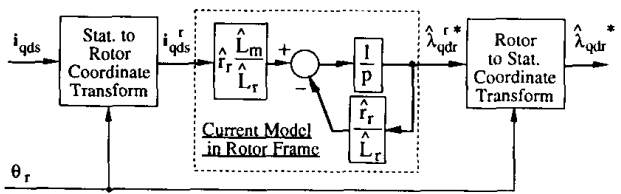

Fig. 20 Current model implemented in the rotor frame, then transformed back into stationary frame for flux reference for closed-loop rotor flux observer.

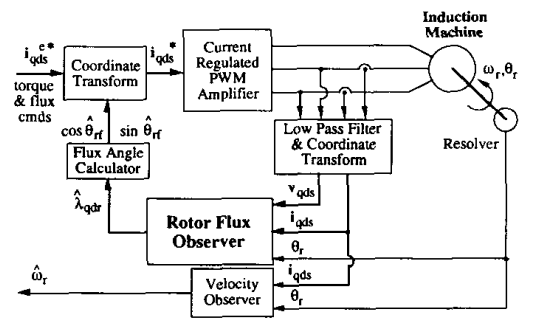

Fig. 21. Direct field oriented controller based upon either open or closed-loop rotor flux observers.

poorly damped eigenvalues at high velocities that can lead to stability problems. Implementation in the rotor frame as shown in Fig. 20 eliminates the cross coupling leaving real eigenvalues at $-\hat{r}_{r} / \hat{L}_{r}$. A second advantage of the rotor frame implementation is that relative rotor position is now required rather than rotor velocity.

Three direct field oriented controllers based upon the two open-loop rotor flux observers, Figs. $2 \& 4$, and the closedloop velocity invariant observer of Fig. 18 were implemented as illustrated in Fig. 21. The observers and coordinate transformations were implemented in software on the Motorola DSP56001 with a $5.33 \mathrm{kHz}$ sample rate. A faster timed interrupt routine sampled and averaged the measured voltages and currents providing an effective integration step of $15.6 \mu \mathrm{sec}$ $(64 \mathrm{kHz})$ [16]. The PWM VSI switched at $3.4 \mathrm{kHz}$ with a stationary frame PI current regulator with $\approx 350 \mathrm{~Hz}$ bandwidth. A 1st order low-pass filter with a $3.9 \mathrm{kHz}$ cut off frequency was used for anti-aliasing. Accurate velocity estimation was obtained via a Luenberger-style velocity observer [19].

Experimental results illustrating the response of the direct field oriented system to a square wave torque command for the three forms of rotor flux estimation are shown in Figs. 22-24. The torque command was toggled between velocity limits. The estimated torque is obtained from the estimated flux and measured stator current, and is thus not equivalent to the true torque developed by the machine. Because the load was dominated by the rotor inertia, the best indicator of system performance is the rotor velocity.

The results generally substantiate the expected behavior. The current model demonstrated a modest sensitivity to the rotor resistance. The voltage model was highly sensitive to the stator resistance at low and zero speeds, but not at higher speeds. Zero speed operation was not possible with detuned stator resistance. The voltage model was also sensitive to the quality and bandwidth of the low pass integrator, as evident by the drift in the flux estimate.

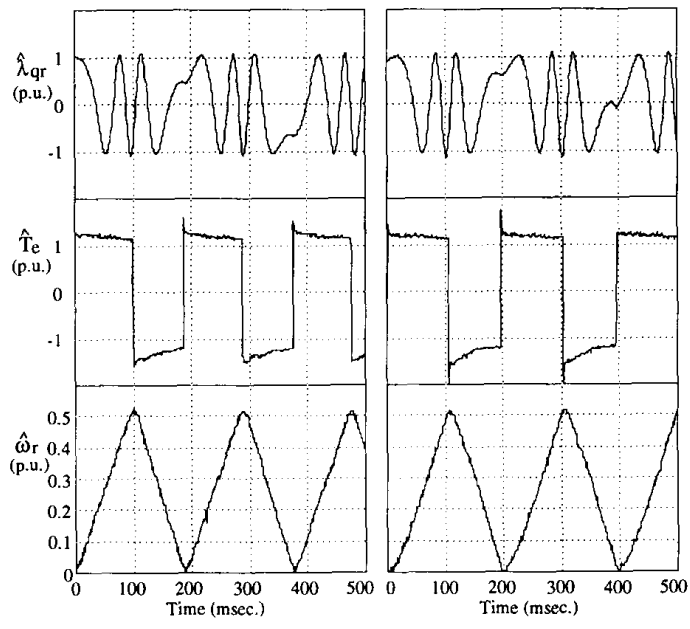

Fig. 22. Rotor flux, torque, and velocity waveforms of tuned and detuned (left is $1 \times r_{r o}$, right is $2 \times r_{r o}$ ) direct field oriented drives based upon the open-loop, current model rotor flux observer.

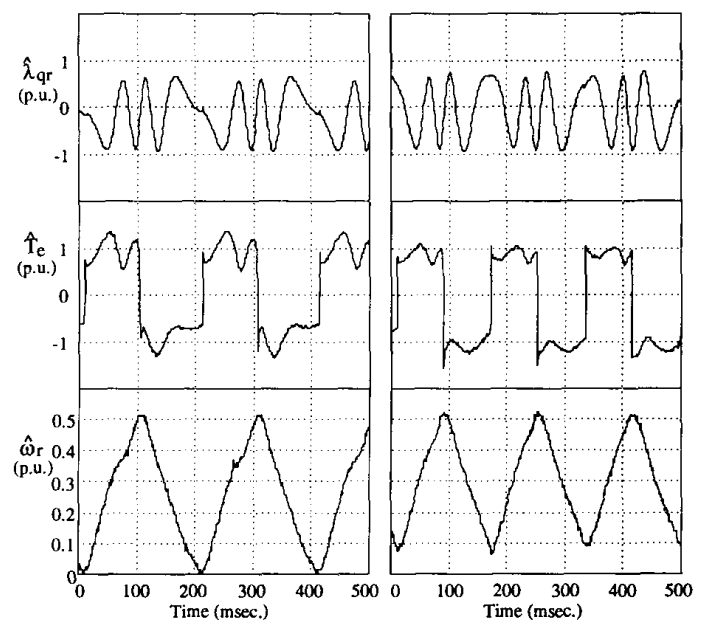

Fig. 23. Rotor flux, torque, and velocity waveforms of tuned and detuned (left is $1 \times r_{s o}$, right is $1.5 \times r_{s O}$ ) direct field oriented drives based upon the open-loop, voltage model rotor flux observer. Detuned zero speed operation not possible.

The closed-loop, velocity invariant observer with current model input provides as good a dynamic result as the current model observer, and substantially better than the voltage model, at velocities below the bandwidth of the observer. At higher velocities the observer moves into the transition region set by the eigenvalues, and then into the voltage model. The low speed integration problems associated with the voltage model are eliminated.

\section{CONCLUSION}

This paper has attempted to contribute the following:

- A clear, physically meaningful accuracy analysis of flux observers. 


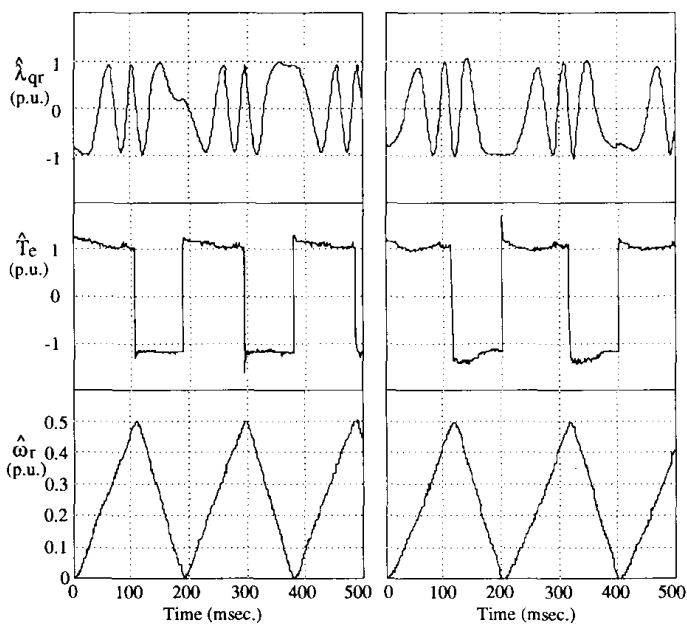

Fig. 24. Rotor flux, torque, and velocity waveforms of tuned and detuned (left is $1 \times r_{r o}, 1 \times r_{s o}$, right is $2 \times r_{r o} .2 \times r_{s o}$ ) direct field oriented drives based upon the closed-loop, velocity invariant rotor flux observer with current model input and two real eigenvalues at 1 and $10 \mathrm{~Hz}$

- A methodology for understanding the source of estimation errors in closed-loop observers, including Gopinath style observers.

- Development of a superior design basis for combining the current and voltage models in a closed-loop observer with velocity invariant dynamics.

- Implementation and experimental evaluation of the flux observer alternatives.

The primary conclusions on the observer topologies are:

- The current and voltage model open loop flux observers and the closed-loop, velocity invariant flux observer all provide flux angular position estimate errors which are relatively small for the $10 \mathrm{hp}$ machine. The net differences in torque characteristics are thus small, except at low frequencies (low speeds) where the current model and the velocity invariant flux observers are superior to the voltage model observer.

- The estimation dynamics and parameter sensitivity of the full order open-loop observer are considerably more complex than the other open loop observers. Like the voltage model, it requires measured voltage input and is thus anticipated to share the zero/low speed implementation problems.

- The cancellation method open-loop estimation method is the least desirable due to its inherent susceptibility to low speed noise and parameter estimate errors.

- The cancellation method acts as an implicit flux reference within both the full order and the Gopinath style closedloop observers.

- The closed-loop, velocity invariant, flux observer with current model input has the desirable low speed attributes of the current model, and the desirable high speed attributes of the voltage model. The bandwidth of the observer establishes the frequency (speed range) of the transition region. Such deterministic properties make this observer ideally suited for wide speed range applications requiring both zero speed and field weakening operation.

\section{APPENDIX}

Induction Machine Parameters Westinghouse TEE II, Frame $215 \mathrm{~T}, 10 \mathrm{Hp}, 3$ phase, $460 / 230 \mathrm{v}, 12.2 / 24.4 \mathrm{~A}, 1750$ rpm, rated $\omega_{s} \approx 0.028$ p.u.

$$
\begin{array}{rlrl}
r_{s} \approx 0.20 \Omega & L_{l s} \approx 1.5 m H & L_{m} \approx 32.3 m H \\
r_{r} \approx 0.20 \Omega & L_{l r} \approx 1.5 m H & J & \approx 0.045 \mathrm{~kg}-\mathrm{m}^{2}
\end{array}
$$

\section{ACKNOWLEDGMENT}

The authors wish to acknowledge the financial support by the National Science Foundation and the technical support and motivation provided by the Wisconsin Electric Machines and Power Electronics Consortium (WEMPEC) of the University of Wisconsin-Madison.

\section{REFERENCES}

[1] D.W. Novotny, and R. D. Lorenz, (eds.), Introduction to Field Orientation and High Performance AC Drives, tutorial book from 1985 \& 1986 IEEE-IAS Annual Meetings.

[2] K. T.Hung, and R. D. Lorenz, "A Rotor Flux Error-Based Adaptive Tuning Approach for Feedforward Field Oriented Machine Drives", Proc. IEEE-IAS Annual Meeting, October 1990.

[3] Moreira, J. C. and Lipo, T. A., "Direct Field Orientation Control Using Third Harmonic Component of the Stator Voltage", International Conference on Electric Machines (ICEM), Boston, MA, August 1990.

[4] Vagati, A. and Villata, F., "A. C. Servo System for Position Control", Proc. ICEM, Lausanne, Switzerland, 1984, pp. 871-874.

[5] Hori, Y., Cotter, V., and Kaya, Y., "A Novel Induction Machine Flux Observer and Its Application to a High Performance AC Drive System", IFAC 10th Triennial World Congress, Munich, FRG, 1987, pp. 363-368.

[6] Verghese, G. C. and Sanders, S. R., "Observers for Flux Estimation in Induction Machine", IEEE Trans. Industrial Electronics, Vol. 35, No. 1. February 1988, pp. 85-94.

[7] Kubota, H. and Matsuse, K., "Flux Observer of Induction Motor with Parameter Adaption for Wide Speed Range Motor Drives", Proc. IPEC Tokyo, 1990, pp. 1213-1218.

[8] Hori, Y, and Umeno, T., "Flux Observer Based Field Orientation (FOFO) Controller for High Performance Torque Control", Proc. IPECTokyo, 1990, pp. 1219-1226.

[9] Franceschini, G., Tassoni, C., and Vagati, A., "Flux Estimation for Induction Servo-Motors", Proc. IPEC-Tokyo, 1990, pp. 1227-1234.

[10] Sangwongwanich, S., Yonemoto, T., Furuhashi, T., and Okuma, S., "Design of Sliding Observer for Robust Estimation of Rotor Flux of Induction Motors", Proc. IPEC-Tokyo, 1990, pp. 1235-1242.

[11] Franceschini, G., Pastorelli, M., Profumo, F., Tassoni, C., and Vagati, A. "About the Gain Choice of Flux Observer in Induction Servomotors", Proc. IEEE-IAS Annual Meeting, 1990, pp. 601-606.

[12] Kubota, H., Matsuse, K., and Nakano, T., "DSP-Based Speed Adaptive Flux Observer of Induction Motor", Proc. IEEE-IAS Annual Meeting, 1991, pp. 380-384.

[13] Böcker, J. and Janning, J., "Discrete-Time Flux Observer for PWM Fed Induction Motors", Proc. EPE, Firenze, 1991, pp. 171-176.

[14] Gopinath, B., "On the Control of Linear Multiple Input-Output Systems", The Bell System Technical Journal, Vol. 50, No. 3, March, 1971, pp. $1063-1081$.

[15] Luenberger, D. G., “An Introduction to Observers", IEEE Trans. on Automatic Control, Vol. AC-16, No. 6, Dec. 1971, pp. 596-602.

[16] Xu, X., De Doncker, R., and Novotny, D. W., "A Stator Flux Oriented Induction Motor Drive", Proc. PESC, Kyoto, April 1988.

[17] Lorenz, R. D. and Lawson, D. B., "Performance of Feedforward, Current Regulators for Field Oriented Induction Machine Controllers", IEEE Trans. Ind. Appl., Vol 1 A-23, No. 2, July/August, 1987, pp. 597-602.

[18] Lorenz, R. D. and Novotny, D. W., "A Controls Systems Perspective of Field Oriented Control for AC Servo Drives", Proc. Controls Engr. Conf., June 6-11, 1988, pp. XVIII - 1 - XVIII-11.

[19] Lorenz, R. D. and Van Patten, K., "High Resolution Velocity Estimation for All Digital, AC Servo Drives", IEEE Trans. Ind. Appl., Vol. 27, No. 4, July/August, 1991, pp. 701-708. 


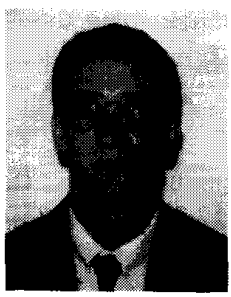

Patrick L. Jansen (S'92) received the B.S. and M.S. degrees in electrical engineering from the University of Wisconsin (UW), Madison, in 1985 and 1987, respectively. He received the Ph.D. degree from UW in December 1993.

From $1987-1989$ he was an experimental scientist in the Commonwealth Scientific and Industrial Research Organization (CSIRO), Division of Applied Physics, New South Wales, Australia, and a development engineer at Machine Dynamics Pty. Ltd. Victoria, Australia.

His thesis research included the integration of electric machine design and transducerless position and velocity estimation, observer-based direct field orientation, and linear induction machine design for high speed material transport systems.
Robert D. Lorenz (S'83-M'84-SM'92) received the B.S., M.S., and Ph.D. degrees, all in mechanical engineering (electro-mechanics and control specialty) from the University of Wisconsin-Madison in 1969, 1970, and 1984, respectively.

Since 1984 he has been a member of the faculty of the University of Wisconsin-Madison, where he is Professor of Mechanical Engineering and of Electrical and Computer Engineering. In this position he acts as Associate Director of the Wisconsin Electric Machines and Power Electronics Consortium and as Co-Director of the Advanced Automation and Robotics Consortium.

He was a Visiting Research Professor in the Electrical Drives Group of the Catholic University of Leuven, Leuven, Belgium and in the Electrical Drives Institute of the Technical University of Aachen, West Germany, in the summers of 1987,1989 , and 1991, respectively. In 1969-1970 he did his Master's thesis research at the Technical University of Aachen, West Germany. From 1972-1982 he was a member of the research staff at the Gleason Works, Rochester, NY. His current research interests include sensor integrated electromagnetic actuator technologies, real time digital signal processing and estimation techniques, and ac drive and high precision machine control technologies.

Dr. Lorenz is a Chairman of the IAS Industrial Drives Committee, and is a member of the Industrial Automation and Control Committee, the Electrical Machines Committee, and the Industrial Automation and Control Committee. He is an active consultant to many organizations and is a Registered Professional Engineer in New York and Wisconsin. He is a member of the ASME, ISA and SPIE. 